\title{
Post-Operative Associated Ocular Status after Cataract Surgery with CTR in Patients with Zonular Dehiscence
}

\author{
Ahmed AHMK ${ }^{1 *}$, Nesa Z
}

\author{
${ }^{1}$ Abu Hena Mostafa Kamal Ahmed, Assistant Professor, Department of Ophthalmology, Shaheed Ziaur Rahman Medical College, \\ Bogura, Bangladesh \\ ${ }^{2}$ Zabun Nesa, Assistant Professor, Department of Ophthalmology, Abdul Malek Ukil Medical College, Naokhali, Bangladesh
}

DOI: $10.36347 /$ sjams.2021.v09i03.030

| Received: 15.12.2020 | Accepted: 26.12.2020 | Published: 23.03.2021

*Corresponding author: Abu Hena Mostafa Kamal Ahmed

Abstract

Original Research Article

Introduction: Surgical management of subluxated cataract presents a challenge to anterior segment surgeons. With recent advances in equipment and instrumentation, better surgical techniques and understanding of fluid dynamics, the surgeon is able to perform relatively safe cataract surgery in the presence of compromised zonules. The CTR could preserve the integrity of the capsular bag, provide the necessary capsular space while giving counter-traction, reduce asymmetric capsular forces, stabilize the vitreous base, facilitate phacoemulsification, aspiration of cortex and IOL implantation, prevent the postoperative IOL decent ration and allow a quicker visual recovery. Aim of the study: To assess the post-operative associated ocular status after cataract surgery with CTR in patients with zonular dehiscence. Material \& Methods: This prospective observational study was conducted at the department of cataract, National Institute of Ophthalmology \& Hospital, Dhaka, during the period of July 2009-December 2009 on cataract patients with zonular dehiscence less than 1800. In this study, 30 cases were included according to the exclusion and inclusion criteria. Sampling technique was non-randomized and purposive. Study patients were explained in details about the disease process, benefits of evaluation, methods and risks of evaluation techniques and then written consent will be taken before conducting the study maintaining the principles of Helsinki declaration. Results: Among 30 patients, age below 40 years was in $14(46.67 \%)$ cases and age $\geq 40$ years was in $16(53.33 \%)$. Mean age was $39.50 \pm 11.59$ (SD) years. Twenty (20) (66.67\%) were male and 10 (33.33\%) were female. Out of 30 cases, 06 (20\%) cases developed corneal oedema which was relieved with treatment; $03(10 \%)$ cases developed mild uveitis which was treated medically with success; $03(10 \%)$ cases developed secondary glaucoma medically managed; 01(3.33\%) case developed retinal detachment managed well with external buckling surgery. Conclusion: Analytical result of the study shows that phacoemulsification with CTR application is an effective and safe surgical procedure for cataract with zonular dehiscence less than $180^{\circ}$. In this study, we recorded very few complications intra and post-operatively which were subsequently managed with good visual outcome.

Keywords: Zonular Dehiscence, Cataract, Outcome, Anterior, Capsular.

Copyright () 2021 The Author(s): This is an open-access article distributed under the terms of the Creative Commons Attribution 4.0 International License (CC BY-NC 4.0) which permits unrestricted use, distribution, and reproduction in any medium for non-commercial use provided the original author and source are credited.

\section{INTRODUCTION}

Surgical management of subluxated cataract presents a challenge to anterior segment surgeons. With recent advances in equipment and instrumentation, better surgical techniques and understanding of fluid dynamics, the surgeon is able to perform relatively safe cataract surgery in the presence of compromised zonules [1]. A capsular tension ring (CTR) may serve a dual purpose, both as a tool providing intraoperative support during cataract removal and as an implant for long-term IOL stabilization. As the diameter of the CTR is larger than that of the capsule bag, the centrifugal forces inherent to the ring expand the capsular equator and buttress the weak areas, providing equal distribution of support over the remaining zonules[2]. The CTR re-expands the capsular bag, provides counter- traction and tautens the posterior capsule during surgery. Jacob et al. [3] showed that phacoemulsification with in-the-bag PC IOL and CTR implantation in eyes with zonular dialysis of up to approximately 150 degrees had a success rate of $90.47 \%$. Visual recovery was not as good as in normal eyes because of the problems associated with zonular dialysis. Finally, they remarked that for the patients of zonular dialysis, the CTR could preserve the integrity of the capsular bag, provide the necessary capsular space while giving counter-traction, reduce asymmetric capsular forces, stabilize the vitreous base, facilitate phacoemulsification, aspiration of cortex and IOL implantation, prevent the postoperative IOL decent ration and allow a quicker visual recovery. The most 
frequent causes of zonular insufficiency that benefit from CTR implantation include pseudo exfoliation, traumatic lens displacement, iatrogenic zonular damage, Marfan's syndrome, homocystinuria, hyper mature cataracts, and post-vitrectomy and filtration patients. The CTR is a useful device that effectively supplements the currently available cataract surgery armamentarium, and develops the application of posterior chamber IOL implantation. During combined cataract and vitreous surgery, a CTR prevents capsule damage and provides undisturbed peripheral visualization before IOL implantation. Capsular tension rings may also influence capsule opacification formation. A special band-shaped CTR with sharp edges was developed to inhibit lens epithelial cell migration and avoid capsulorhexis-optic contact. Dorecka et al. [4] evaluated long term results of phacoemulsification with PC IOL and capsular tension ring (CTR) implantation in lens subluxation. The study comprised of 134 patients-146 eyes with subluxated lens. In all cases phacoemulsification with PC IOL and CTR implantation was performed. No intraoperative complications have occurred. Postoperative complications included inflammation in the anterior chamber in three eyes $(2.1 \%)$, retinal detachment in 2 eyes $(1.4 \%)$. In all cases, there was no PC IOL decent ration. They remarked that (1) CTR facilitates phacoemulsification with PC IOL implantation in lens subluxation. (2) Phacoemulsification of subluxated lens with PC IOL and CTR implantation seems to be safe and effective procedure.

\section{Methodology and Materials}

This prospective observational study was conducted at the department of cataract, National Institute of Ophthalmology \& Hospital, Dhaka, during the period of July 2009-December 2009 on cataract patients with zonular dehiscence less than 1800 . In the study, 30 cases were included according flowing exclusion and inclusion criteria. Sampling technique was non-randomized and purposive. We followed the closed chamber technique, which was achieved only by phacoemulsification. Our surgical paradigms included a 3-plane valvular incision, injection of viscoelastic in anterior chamber before removing any instrument from the eye, bimanual irrigation and aspiration and 2-port anterior chamber vitrectomy. Superior-temporal location was preferred irrespective of the site of subluxation. Before initiating capsulorhexis the anterior chamber was deepened with injection viscoelastic. A side entry was made at 2 and $3 o^{\prime}$ clock positions away from the main incision using a 15-degree paracentesis knife. An initial small anterior capsulorhexis was attempted and then a definitive large rhexis (I \& D rhexis) was performed after capsulorhexis and before hydro dissection. After capsulorrhexis, CTR $12.0 \mathrm{~mm}$ diameter ring (Morcher type 14) was introduced. Then, thorough, gentle but careful multi-quadrant hydro dissection was performed. Phacoemulsification was performed using power appropriate for the grade of cataract, low vacuum and aspiration setting with minimal bottle height. After phacoemulsification in bag, PCIOL was implanted in all cases. Data were collected in a pre-designed data collection sheet. Data were analyzed by appropriate tests. A probability ' $p$ ' value of 0.5 or less was considered as statistically significant. Study patients were explained in details about the disease process, benefits of evaluation, methods and risks of evaluation techniques and then written consent will be taken before conducting the study maintaining the principles of Helsinki declaration.

- Inclusion Criteria: Patients with

- Either sex

- Zonular dehiscence $<180^{\circ}$

- Very hard cataract having the risk of intra-operative zonular dehiscence.

- Exclusion Criteria: Patients with zonular dehiscence more than $180^{\circ}$, corneal opacity, shallow A/C.

\section{RESULTS}

Among 30 patients, age below 40 years was in $14(46.67 \%)$ cases and age $\geq 40$ years was in 16 $(53.33 \%)$. Mean age was $39.50 \pm 11.59$ (SD) years (Figure I). Table I shows the distribution of sex among study subjects. Twenty (20) (66.67\%) were male and 10 $(33.33 \%)$ were female. Out of 30 cases, $14(46.67 \%)$ were traumatic subluxation and $16(53.33 \%)$ were nontraumatic subluxation. Among non-traumatic subluxations, $09(30 \%)$ were hereditary ectopia lentis, $04(13.33 \%)$ were pseudo exfoliation syndromes and 03 $(10 \%)$ were hypermature cataract related (Table II). Out of 30 cases, 06 (20\%) cases developed corneal oedema which was relieved with treatment; $03(10 \%)$ cases developed mild uveitis which was treated medically with success; $03(10 \%)$ cases developed secondary glaucoma medically managed; 01(3.33\%) case developed retinal detachment managed well with external buckling surgery. IOL drop in the vitreous occurred in 01 case, which was managed by pars plane vitrectomy, IOL removal and scleral fixation IOL implantation with good post-operative vision. Six (6) $(20 \%)$ cases developed mild to moderate degree of decent ration that was managed optically. Mild posterior capsular opacity (PCO) developed in 03 $(10 \%)$ cases (Table III). 


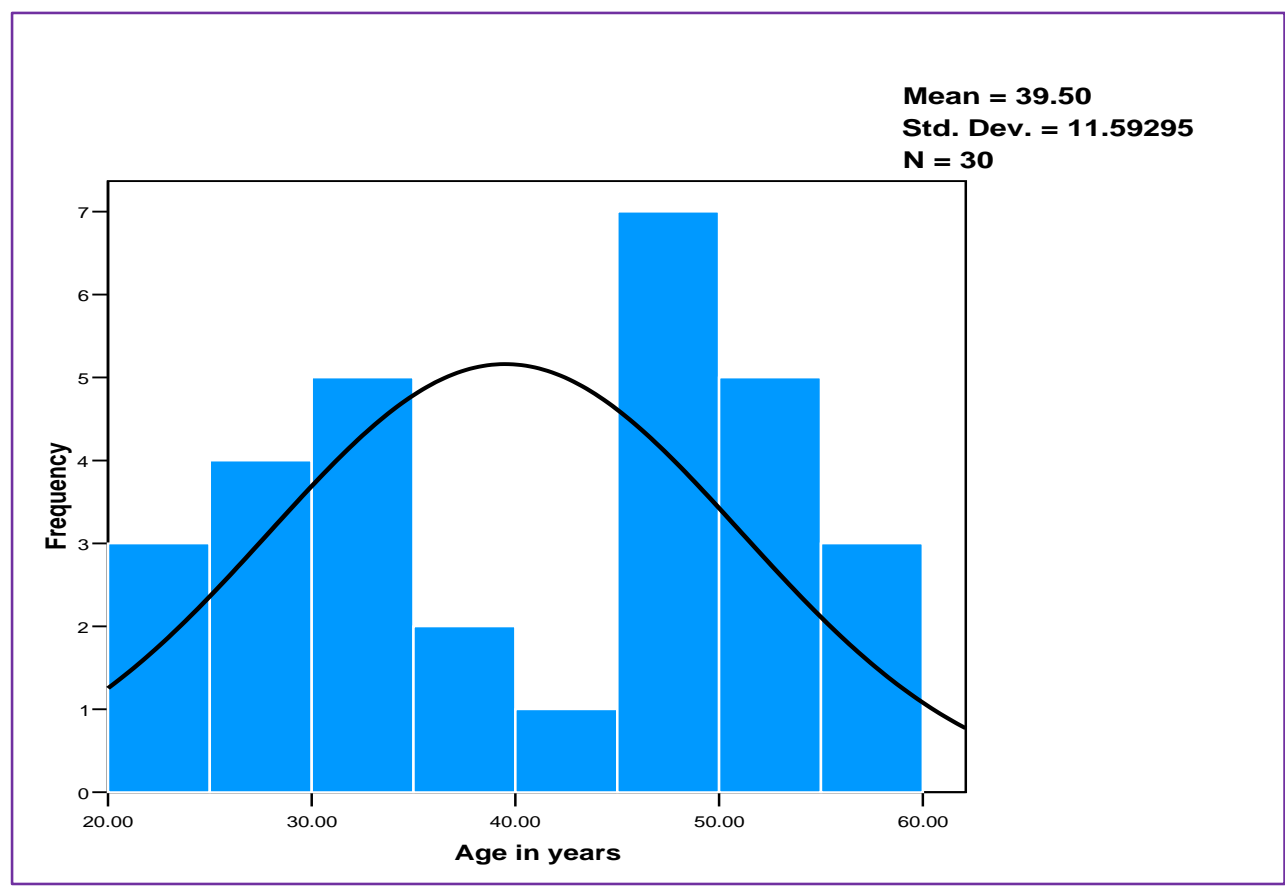

Fig-I: Histogram showing age distribution of study subjects

Table-I: Distribution of sex among study subjects

\begin{tabular}{|l|l|}
\hline Sex group & No. of patient (\%) \\
\hline Male & $20(66.67)$ \\
\hline Female & $10(33.33)$ \\
\hline
\end{tabular}

Table-II: Distribution of causes of lens subluxation

\begin{tabular}{|l|c|}
\hline Causes of subluxation & No. of patient (\%) \\
\hline Traumatic & $14(46.67)$ \\
\hline Non-traumatic: & $16(53.33)$ \\
Hereditary ectopia lentis & $09(30)$ \\
Pseudoexfoliation syndrome & $04(13.33)$ \\
Hypermature cataract & $03(10)$ \\
\hline
\end{tabular}

Table-III: Distribution of post-operative profile

\begin{tabular}{|l|l|l|}
\hline V/A & $\begin{array}{l}\text { Pre-operative } \\
\text { No. of patient }(\%)\end{array}$ & $\begin{array}{l}\text { Post-operative } \\
\text { No. of patient }(\%)\end{array}$ \\
\hline $6 / 12$ or better & 00 & $09(30)$ \\
\hline $6 / 18-6 / 24$ & 00 & $12(40)$ \\
\hline $6 / 36$ & $03(10)$ & $03(10)$ \\
\hline $6 / 60$ & $04(13.33)$ & $02(6.67)$ \\
\hline $5 / 60-3 / 60$ & $09(30)$ & $03(10)$ \\
\hline$<3 / 60-$ Hand motion & $14(46.67)$ & $01(3.33)$ \\
\hline
\end{tabular}

\section{DiscuSsiON}

Among 30 patients, age below 40 years was in $14(46.67 \%)$ cases and age $\geq 40$ years was in 16 $(53.33 \%)$. Mean age was $39.50 \pm 11.59$ (SD) years. Praveen et al. [5] 14 reported that mean age was 39.15 +/- 16.33 (range 5-74). Trauma appears more in relatively younger age. Congenital ectopia lentis often manifest after $2^{\text {nd }}$ to $3^{\text {rd }}$ decade. That is the reason average age in the current study was around 40 years, which is comparable with other relevant studies. Out of 30 subjects $20(66.67 \%)$ were male and $10(33.33 \%)$ were female. Males appear more prone to ocular trauma than females. Again, traumatic sub-luxation is the commonest cause. Therefore, a male preponderance has been reported by many studies [6-9]. Praveen et al.[5] reported $75 \%$ male and $25 \%$ Out of 30 cases, 14 $(46.67 \%)$ were traumatic subluxation and $16(53.33 \%)$ were non-traumatic subluxation. Among non-traumatic sub-luxations, $09(30 \%)$ were hereditary ectopia lentis, $04(13.33 \%)$ were pseudo-exfoliation syndromes and 03 $(10 \%)$ were hypermature cataract related. Praveen et al. [5] evaluated the outcome of phacoemulsification in eyes with subluxated cataract. This retrospective study comprised 22 eyes of 20 consecutive patients with 
Ahmed AHMK \& Nesa Z; Sch J App Med Sci, Mar, 2021; 9(3): 445-449

subluxated cataracts of varying aetiology. They found that traumatic subluxation was the commonest $(55 \%)$. Out of 30 cases, 06 (20\%) cases developed corneal oedema which was relieved with treatment; $03(10 \%)$ cases developed mild uveitis which was treated medically with success; $03(10 \%)$ cases developed secondary glaucoma medically managed; 01(3.33\%) case developed retinal detachment managed well with external buckling surgery. IOL drop with CTR dislocation in the vitreous occurred in 01 case, which was managed by pars plana vitrectomy, IOL and CTR removal and scleral fixation IOL implantation with good post-operative vision afterwards. Six (6) (20\%) cases developed mild to moderate degree of decentration that was managed optically. Mild posterior capsular opacity (PCO) developed in $03(10 \%)$ cases. Praveen et al. [5] reported rise in IOP in one eye $(4.54 \%)$ and nine eyes $(40.9 \%)$ had geometrically decentered IOLs. Jacob et al. [3] studied that there was minimal corneal edema in 2 eyes $(9.52 \%)$ and mild iritis in 5 eyes $(23.80 \%)$. Three eyes $(14.28 \%)$ developed raised intraocular pressure that responded well to medical therapy. Tribus et al. [10] reported five eyes (5\%) having a slight dislocation of the IOL noted postoperatively, but none of these patients complained of visually relevant symptoms. Lee et al. [11] reported their findings on 40 eyes of 20 patients who were followed for 2 months. Each patient had an IOL in one eye and an IOL with a CTR in the fellow eye. Comparatively, the IOL-CTR group had a statistically lower rate of IOL decent ration compared with the IOLonly group using Scheimpflug image analysis. The mean decent ration in the IOL-CTR group was $0.42 \pm$ $0.17 \mathrm{~mm}$, whereas in the IOL-only group it was $0.57 \pm$ $0.16 \mathrm{~mm}$. Although the incidence of PCO is reduced with the use of CTRs [12], PCO has still been reported after surgery [13]. To minimize the risk of PCO, Nishi et al.'s [14] capsular bending ring (CBR) may be utilized, with the added feature of a square edge. This model has been shown to significantly reduce the risk of posterior capsule epithelial growth. In additional, Dick et al. [15] reported that combining a viscoadaptive viscoelastic agent and a CBR not only enhances the safety of primary and secondary PCIOL implantation and IOL exchange in pediatric cases, but also reduces PCO. A square-edged IOL design used in conjunction with a CTR may also decrease the incidence of PCO [16].

\section{Limitations OF THE STUdy}

1. Study period was very short.

2. Sample size was comparatively small.

\section{Conclusionand}

\section{RECOMMENDATIONS}

Analytical result of the study shows that phacoemulsification with CTR application is an effective and safe surgical procedure for cataract with zonular dehiscence less than $180^{\circ}$. In this study, we recorded very few complications intra and postoperatively which were subsequently managed with good visual outcome. Causes of poor visual outcome were pre-existing posterior segment anomalies. Further prospective study with larger sample and longer follow up period is recommended.

\section{Funding}

$$
\text { No funding sources }
$$

\section{Conflict of interest}

None declared

\section{Ethical approval}

The study was approved by the Institutional Ethics Committee

\section{REFERENCES}

1. Lu Lw, Fine IH. Phacoemulsification in difficult and challenging cases: Phacoemulsification in subluxated Cataract. New York, Stuttgart: Thieme. 1999: 99-110.

2. Leger U. MD, Witschel BM, MD, Lim SJ, MD. 'The Capsular Ring: A New Device for Complicated Cataract Surgery', film presented at the $3^{\text {rd }}$ American-International Congress on Cataract, IOL and Refractive Surgery, Seattle, Washington, USA, May 1993.

3. Jacob S, Agarwal A, Agarwal A. Efficacy of a capsular tension ring for phacoemulsification in eyes with zonular dialysis. J Cataract Refract Surg. 2003; 29:315-321.

4. Dorecka M, Rokicki W, Nita M, Krysik K, Nita E, Sikorska A, Romaniuk W. Phacoemulsification of subluxated lens with capsular tension ring implantation. Klin Oczna. 2007; 109(7-9):280-2.

5. Praveen MR, Vasavada AR, Singh R. Phacoemulsification in subluxated cataract. Indian Journal of ophthalmology. 2003 Jun 1;51(2):147.

6. Parrish RK II. Anatomy, physiology, and pathology of the crystalline lens. In: Bascom Palmer Eye Institute's Atlas of Ophthalmology. 1999:241.

7. Albert DM, Jakobiec FA. Pathology of the lens. In: Principles and Practice of Ophthalmology. 2000:2225-2239.

8. Nelson LB, Maumenee IH. Ectopia lentis. Surv Ophthalmol. Nov-Dec 1982; 27(3):143-60.

9. Nirankari MS, Chaddah MR. Displaced lens. Am J Ophthalmol. Jun 1967; 63(6):1719-23.

10. Tribus C, Alge CS, Haritoglou C, Lackerbauer C, Kampik A, Mueller A, Priglinger SG. Indications and clinical outcome of capsular tension ring (CTR) implantation: A review of 9528 cataract surgeries. Clin Ophthalmol.2007 March; 1(1): 6569.

11. Lee DH, Shin SC, Joo CK. Effect of a capsular tension ring on intraocular lens decentration and tilting after cataract surgery. J Cataract Refract Surg. 2002; 28:843-846. 
12. De' liseo D, Longanesi L, Grisanti F, Negrini V. Prevention of posterior capsule opacification using capsular tension ring for zonular defects in cataract surgery. Eur J Ophthalmol. 2003; 13(2):151-154.

13. Ahmed IK, Butler M. Capsular Tension Devices for the Glaucoma Surgeon. In: Glaucoma Today. Nov-Dec 2004: 1-4.

14. Nishi O, Nishi K, Menapace R. Capsule-bending ring for the prevention of capsular opacification: a preliminary report. Ophthalmic Surg Lasers. 1998; 29:749-753.
15. Dick HB, Schwenn O, Pfeiffer N. Implantation of the modified capsular bending ring in pediatric cataract surgery using a viscoadaptive viscoelastic agent. J Cataract Refract Surg. 1999; 25:14321436.

16. Saco S, Menapace R, Findl O. Long-term efficacy of adding a sharp posterior optic edge to a threepiece silicone intraocular lens on capsule opacification: five-year results of a randomized study. Am J Ophthalmol. 2005; 139:696-703? 